\title{
Avaliação diagnóstica na síndrome disfunção cognitiva canina
}

\author{
[Diagnostic Evaluation of Canine Cognitive Dysfunction Syndrome]
}

\author{
F.D.M. Krug ${ }^{l}$, M.T. Tillmann ${ }^{2}$, M.B.C. Piñeiro ${ }^{l}$, C.B.M. Mendes ${ }^{l}$, \\ S.O. Capella ${ }^{1}$, F.R.P. Bruhn ${ }^{3}$, M.O. Nobre ${ }^{3}$ \\ ${ }^{1}$ Pós-graduação - Universidade Federal de Pelotas - Pelotas, RS \\ ${ }^{2}$ Universidade do Oeste de Santa Catarina - Xanxerê, SC \\ ${ }^{3}$ Universidade Federal de Pelotas, RS
}

\begin{abstract}
RESUMO
O objetivo do presente estudo foi avaliar o questionário observacional e os testes de reatividade como forma de triagem e diagnóstico da disfunção cognitiva em cães idosos. Foram estudados 10 cães acima de sete anos, que apresentavam queixas comportamentais. Foi utilizado questionário que abordava questões comportamentais, como desorientação, atividade, interação socioambiental, alterações no padrão do sono e casa-sujidade. As respostas foram convertidas em pontuações, cujo somatório classificou o cão com disfunção cognitiva canina (DCC), ou borderline (BL), ou sem alterações comportamentais (SAC). Logo depois, foram realizados, em todos os cães, os seguintes testes cognitivos: open field, curiosidade, interação com humano e com espelho. Pela avaliação do questionário, foi determinado que dois cães tinham DCC, três eram BL, cinco eram SAC. Os cães classificados com DCC tinham idade superior aos demais e apresentaram alterações em todos os testes de reatividade, enquanto os cães BL apresentaram alterações em dois testes de reatividade e os SAC não apresentaram alterações. Conclui-se que, com o aumento da expectativa de vida canina, o questionário observacional foi um instrumento de triagem para a identificação dos cães classificados com DCC, BL e SAC, e os testes de reatividade como um método inovador para identificar o verdadeiro estado cognitivo dos pacientes idosos.
\end{abstract}

Palavras-chave: senilidade, déficit cognitivo, open field, curiosidade, interação com humano

\begin{abstract}
The objective was to evaluate the observational questionnaire and the reactivity tests as a way of screening and diagnosis of cognitive dysfunction in elderly dogs. Ten dogs over seven years of age, with behavioral complaints, were studied. A questionnaire was used that addressed behavioral issues such as disorientation, activity, socioenvironmental interaction, changes in sleep pattern, and house-dirtiness. Responses were converted into scores, which summed the dog with canine cognitive dysfunction (DCC), or Borderline (BL) or without behavioral changes (SAC). Soon after, the following cognitive tests were performed on all dogs: open field, curiosity, interaction with human and with mirror. Through questionnaire evaluation, two dogs had CHD, three were BL, and five were SAC. The dogs classified with DCC were older than the others and presented alterations in all reactivity tests, while the BL dogs presented changes in two reactivity tests and CAD showed no alterations. With the increase in canine life expectancy, the observational questionnaire was a screening instrument for the identification of dogs classified with DCC, BL and SAC and the reactivity tests as an innovative method to identify the true cognitive status of the dogs elderly patients.
\end{abstract}

Keywords: senility, cognitive impairment, open field, curiosity, interaction with human

\section{INTRODUÇÃO}

A disfunção cognitiva canina (DCC) é uma síndrome neurodegenerativa semelhante à doença de Alzheimer em humanos e em ambas o

Recebido em 12 de julho de 2017

Aceito em 26 de março de 2018

E-mail: fernandamktug@gmail.com diagnóstico definitivo só é confirmado post mortem (Yu et al., 2011; Pineda et al., 2014). No entanto, na busca de um diagnóstico in vivo, temse procurado utilizar critérios clínicos para se determinar o declínio cognitivo por meio de testes neuropsicológicos. Na rotina de clínica de 
pequenos animais, quando há queixa de sinais que indicam uma possível disfunção cognitiva, como desorientação, alterações do sono, déficit de memória e aprendizagem, deve-se iniciar a avaliação do paciente, mediante uma anamnese detalhada, avaliando-se o comportamento por meio de questionário observacional e de testes de reatividade (Tapp et al., 2004; González et al., 2012). O questionário observacional é uma ferramenta válida como teste de triagem, que possibilita a avaliação da disfunção cognitiva e da sua progressão, assim como permite observar a resposta ao tratamento (Schütt et al., 2015; Madari et al., 2015). Enquanto os testes de reatividade são instrumentos importantes para avaliar com maior precisão o estado cognitivo dos cães e monitorar a progressão da síndrome (Rosado et al., 2012a). Estudos recentes têm sido desenvolvidos para avaliar e comprovar o declínio cognitivo de cães, causado progressivamente pelo envelhecimento (González et al., 2012; Pineda et al., 2014), inclusive demonstrando que cães idosos apresentam aprendizagem mais lenta, enquanto a memória a longo prazo é preservada (Wallis et al., 2016).

No Brasil, foram realizados testes de reatividade, como: abordagem à recompensa, ao objeto, aprendizado reverso, atraso não ligado à posição e atraso não ligado à amostra, para avaliar a memória e aprendizagem de cães (Heckler et al., 2014). Esses testes permitem uma avaliação detalhada do estado cognitivo do cão, mas são difíceis de serem aplicados na rotina clínica, por necessitarem de profissionais treinados e materiais específicos; os animais avaliados precisam apresentar um temperamento estável e, ainda, o tempo de duração da aplicação dos testes é de aproximadamente 10 dias. Porém, existem outros testes considerados mais simples e facilmente aplicáveis na rotina clínica, que podem auxiliar na identificação da síndrome de disfunção cognitiva canina em qualquer fase, ao avaliarem a locomoção, o comportamento e a interação social dos animais, por meio dos testes de open field, curiosidade, interação com humanos e espelho (Martinez et al., 2013).

Diante do aumento da expectativa de vida dos cães, procura-se diagnosticar, de forma precoce, as patologias relacionadas às disfunções cognitivas relacionadas à senilidade. Assim, busca-se maior qualidade de vida aos cães, mediante tratamento adequado para reduzir a progressão da síndrome e melhorar a condição de vida dos animais idosos e a relação com seus tutores (Head et al., 2002). Considerando que a avaliação cognitiva de cães idosos deve ser realizada preventivamente em todos os cães com mais de sete anos de idade, por meio de um método aplicável na rotina clínica, foi desenvolvido este estudo, com o objetivo de avaliar o questionário observacional e os testes de reatividade como forma de triagem $\mathrm{e}$ diagnóstico da síndrome de disfunção cognitiva em cães idosos.

\section{MATERIAL E MÉTODOS}

O estudo foi aprovado no Comitê de Ética e Experimentação Animal da Universidade Federal de Pelotas, pelo número 4655-2015. Para o estudo, os cães foram selecionados aleatoriamente, sendo incluídos aqueles que tinham acima de sete anos de idade, apresentavam queixas comportamentais e cujos tutores se disponibilizaram a responder o questionário observacional e autorizaram a realização dos testes de reatividade. Como critério de exclusão, foram descartados do estudo os animais que apresentavam alterações clínicas agudas e/ou hematológicas.

As queixas foram agrupadas em cinco categorias: alterações nas atividades do dia a dia, desorientações em relação à mobilidade e para a eliminação de excreções (evacuar ou urinar), dificuldades para interação socioambiental e alterações no ciclo sono e vigília. Para alterações relacionadas às atividades, foram consideradas as seguintes queixas: anda sem parar; anda sem rumo aparente; late sem motivo aparente; tem apetite voraz; parece incapaz de ouvir sons baixos. Nas alterações de desorientação, considerou-se ficar preso entre os móveis e/ou objetos da casa. Para as alterações relacionadas à eliminação de excreções, foi considerado o ato de urinar e/ou defecar em locais que naturalmente o cão não o faria, como no local de dormir. Nas alterações referentes à interação socioambiental, considerou-se a agressividade com outros animais e/ou com pessoas e também aqueles casos em que havia uma ligação extrema do cão com o tutor ou, ao contrário, quando não havia interação com o tutor. Por fim, foram consideradas alterações do sono e vigília quando ocorria inquietação durante o sono ou quando o 
animal dormia mais durante o dia do que durante a noite.

Após a indicação das queixas principais, os tutores responderam ao questionário observacional adaptado de Osella et al. (2007) e Regenerative Neuroscience Group (Canine..., 2012), que contém categorias relacionadas ao comportamento do cão. $\mathrm{O}$ resultado do questionário observacional foi expresso pelo somatório final das respostas; assim, cães com somatório até 39 pontos foram considerados SAC (sem alterações consideráveis), de 40 a 49 pontos BL (borderline), e cães com somatório de 50 pontos ou mais foram considerados DCC (disfunção cognitiva canina) (Canine, 2012). Os cães foram numerados em ordem crescente, de acordo com a pontuação obtida no questionário observacional.

$\mathrm{Na}$ sequência, realizaram-se os seguintes testes de reatividade: open field, curiosidade, interação com humano e do espelho, sendo adaptados de Rosado et al. 2012b. Os testes foram feitos em local demarcado, em área fechada de $32,4 \mathrm{~m}^{2}$, tendo como piso tatames, e, para a realização das filmagens, foi instalada uma câmera (Samsung, modelo WB150F) a dois metros de altura. Os testes foram filmados e tiveram duração de 32 minutos/cão, sendo cada parâmetro avaliado durante três minutos, com um intervalo de cinco minutos entre cada teste para que o cão descansasse.

No teste chamado open field (OF), ou de campo aberto, colocou-se o cão na sala, a fim de se avaliar o comportamento de locomoção (normal ou marcha estereotipada) e a exploração do ambiente pela manifestação de condutas de olfato, micção e vocalização. No teste de curiosidade (TC), foram colocados três brinquedos diferentes (pote, bolinha e halter) no centro da sala, para se avaliar a interação e o interesse do cão em relação aos objetos diferentes. Já no teste de interação com humano (TIH), uma pessoa desconhecida sentou-se no centro da sala, ignorou completamente o cão e permaneceu imóvel, momento em que o animal foi avaliado quanto à busca ou não pela interação com o humano. No teste do espelho (TE), verificou-se a interação do animal perante a própria imagem refletida.
Nos vídeos, foram avaliados se havia distúrbio (presença ou ausência) para cada um dos parâmetros avaliados: interação com objetos novos e com pessoas, além da locomoção (normal ou marcha estereotipada), condutas de vocalização, eliminação de excreções, olfação e a atitude do cão diante do reflexo da própria imagem no espelho. Foi realizada uma avaliação intra-avaliadores (três), os quais desconheciam o assunto, sendo os dados selecionados de acordo com o predomínio de concordância entre eles.

Os resultados dos testes de reatividade foram associados à classificação do questionário em DCC, BL e SAC, e cruzados por meio de testes de qui-quadrado em software estatístico Statistic 9.0. Considerou-se um nível mínimo de confiança de $95 \%$ em todas as análises estatísticas.

\section{RESULTADOS E DISCUSSÃO}

Este estudo demonstrou que os questionários observacionais aliados aos testes de reatividade são ferramentas úteis para avaliar pacientes senis com suspeita de déficit cognitivo, pois auxiliam o clínico a identificar, de maneira precoce, os primeiros sinais comportamentais compatíveis com a síndrome de disfunção cognitiva canina. Isso permite o tratamento medicamentoso, nutricional e ambiental, o que promove uma melhor qualidade de vida dos cães idosos e, consequentemente, a relação deles com seus tutores.

Para a realização do estudo, foram avaliados 10 cães de porte variável, de ambos os sexos, com idade entre oito e 18 anos. No questionário observacional, os resultados foram: cinco $(50 \%)$ cães classificados como SAC, três (30\%) classificados com BL e dois $(20 \%)$ classificados com DCC (Tab. 1). Os cães com DCC tinham nove e 18 anos, ambos idosos e com desenvolvimento natural da DCC. Salienta-se que cães com epilepsia idiopática apresentam maior risco de desenvolvimento precoce da DCC, quando comparados a cães sem esta afecção, o que aumenta naturalmente o risco do desenvolvimento da DCC, conforme vão envelhecendo (Packer et al., 2018). Os dois cães com DCC deste estudo eram de porte médio e grande, sendo ambas fêmeas. Os estudos não têm demonstrado predisposição para o desenvolvimento do DCC em relação ao sexo e 
ao porte dos cães (Fast et al., 2013; Katina et al., 2016), embora em cães mais idosos (11-13 anos de idade) tenha sido demonstrado diferença estatística em relação ao porte médio e grande (Katina et al., 2016). Ainda, as fêmeas e os machos castrados podem ter maior predisposição em razão do declínio dos hormônios sexuais (Landesberg, 2012).

Alguns tutores relatam mais de uma queixa comportamental (Tab. 1); as mais frequentes foram atividade (40\%) e interação socioambiental $(30 \%)$. E as menos relatadas foram alterações na desorientação (20\%) e casa/sujidade (10\%). Em relação aos resultados obtidos em outros estudos com questionário observacional, foi demonstrado que o comprometimento na interação social foi mais frequente em cães com comprometimento cognitivo leve (40\%) e moderado (67\%); nestes ultimos, afetando também a interação social e o ciclo sono-vigília (Rofina et al., 2006). Já naqueles com disfunção cognitiva grave, houve uma tendência a apresentar comprometimento de todos os quatro domínios estudados (orientação espacial, interações sociais, ciclos de sono-vigília e sujidade em casa) (Madari et al., 2015).

Tabela 1. Demonstração da condição cognitiva de pacientes caninos senis por meio do questionário observacional e da avaliação dos testes de reatividade em: com alteração (+) e sem alteração (-)

\begin{tabular}{|c|c|c|c|c|c|c|c|c|c|c|c|}
\hline \multirow{2}{*}{ Cão } & \multirow{2}{*}{ Sexo } & \multirow{2}{*}{$\begin{array}{l}\text { Idade } \\
\text { (anos) }\end{array}$} & \multirow{2}{*}{ Raça } & \multirow{2}{*}{ Porte } & \multirow{2}{*}{$\begin{array}{c}\text { Queixa } \\
\text { comportamenta } \\
1\end{array}$} & \multicolumn{2}{|c|}{ Questionário observacional } & \multicolumn{4}{|c|}{ Teste de reatividade } \\
\hline & & & & & & Pontos & Classificação & TOF & $\mathrm{TC}$ & TIH & $\mathrm{TE}$ \\
\hline 1 & M & 14 & SRD & Médio & Desorientação & 36 & SAC & - & - & - & - \\
\hline 2 & $\mathrm{~F}$ & 13 & SRD & Grande & Atividade & 38 & SAC & - & - & - & - \\
\hline 3 & $\mathrm{~F}$ & 13 & SRD & Médio & Atividade & 28 & SAC & - & - & - & - \\
\hline 4 & $\mathrm{~F}$ & 10 & CRD & Médio & Atividade & 39 & SAC & - & - & - & - \\
\hline 5 & $\mathrm{~F}$ & 10 & SRD & Pequeno & $\begin{array}{c}\text { Interação } \\
\text { socioambiental }\end{array}$ & 36 & SAC & - & - & - & - \\
\hline 6 & $\mathrm{~F}$ & 11 & CRD & Pequeno & $\begin{array}{c}\text { Interação } \\
\text { socioambiental }\end{array}$ & 40 & $\mathrm{BL}$ & + & + & - & - \\
\hline 7 & M & 9 & SRD & Médio & $\begin{array}{c}\text { Interação } \\
\text { socioambiental } \\
\text { Desorientação }\end{array}$ & 45 & $\mathrm{BL}$ & - & + & + & - \\
\hline 8 & $\mathrm{~F}$ & 8 & CRD & Pequeno & $\begin{array}{c}\text { Interação } \\
\text { socioambiental }\end{array}$ & 47 & $\mathrm{BL}$ & + & + & + & - \\
\hline 9 & $\mathrm{~F}$ & 18 & SRD & Médio & Atividade & 54 & DCC & + & + & + & + \\
\hline 10 & $\mathrm{~F}$ & 9 & CRD & Gigante & $\begin{array}{c}\text { Interação } \\
\text { socioambiental }\end{array}$ & 51 & DCC & + & + & + & + \\
\hline & & & & & Casa/sujidade & & & & & & \\
\hline
\end{tabular}

$\mathrm{M}=$ macho; $\mathrm{F}=$ fêmea; $\mathrm{SRD}=$ sem raça definida; $\mathrm{CRD}=$ com raça definida; $\mathrm{SAC}=$ sem alterações consideráveis; $\mathrm{BL}=$ borderline $; \mathrm{DCC}=$ disfunção cognitiva canina; positivo $=(+)$; negativo $=(-) ; \mathrm{TOF}=$ teste open field ;C $=$ teste de curiosidade; $\mathrm{TIH}=$ teste de interação com humanos; $\mathrm{TE}=$ teste do espelho.

Observou-se que os cães classificados do grupo DCC apresentaram alterações em todos os testes de reatividade (Tab. 1), corroborando a disfunção cognitiva. Já os cães BL apresentavam alterações em pelo menos dois testes de reatividade (Tab.
1), demonstrando, assim, uma tendência à alteração na função cognitiva, enquanto os cães SAC não demonstraram alterações em nenhum dos testes de reatividade (Tab.1), possivelmente por esses animais apresentarem alterações 
comportamentais e não cognitivas (Siwak et al., 2003). Isso mostra que a associação do questionário observacional com os testes de reatividade em cães idosos é válida, para distinguir alterações cognitivas e comportamentais, sendo relevante especialmente para o clínico veterinário, pois o diagnóstico diferencial dessa síndrome é complexo e tem uma casuística elevada nos dias atuais (Colle et $a l .$, 2000). Assim, a utilização do questionário observacional e dos testes de reatividade precisa estar incluída nas avaliações iniciais de cães idosos, como triagem, para definir a presença de alterações cognitivas que devem, na sequência, ser investigadas com o uso de exames mais complexos, como a tomografia computadorizada (Pineda et al., 2014). Ainda, é importante a análise rotineira de cães maduros/idosos, já que foi demonstrado que alguns cães que eram BL progrediram para DCC após algum tempo; portanto, existe o risco prospectivo dessa evolução (Fast et al., 2013).

No teste open field (Fig. 1A), a maioria dos cães $(1,2,4,5,6,7,8$, e 10) locomoveu-se normalmente pela sala, com exceção do cão número três, que não se locomoveu, e do número nove, que apresentou a marcha estereotipada, alteração compatível de cães que apresentam a síndrome de disfunção cognitiva canina. Nos cães com DCC, a maior frequência da apresentação do comportamento de não locomoção, em que ficam presos atrás de objetos, sem contorná-lo, foi relacionada com o aumento de outras alterações comportamentais (Salvin et al., 2011; Rosado et al., 2012a).

Durante o teste de reatividade, os cães classificados no grupo DCC, pelo questionário, apresentaram uma marcha estereotipada e caminharam sem rumo aparente. A desorientação, a alteração do ciclo do sono, a diminuição da interação e a ansiedade, juntas, são os principais sinais apresentados pelos cães com DCC (Fast et al., 2013). Essa alteração de comportamento provavelmente acontece em razão das alterações atribuídas à atrofia cerebral e ao acúmulo da proteína beta-amiloide, que forma as chamadas placas senis na região do córtex pré-frontal e no hipocampo (Colle et al., 2000). Da mesma forma que em humanos com a doença de Alzheimer, em cães o acúmulo de proteína beta-amiloide está diretamente relacionado ao declínio cognitivo (Fast et al.,
2013). Por isso, cada vez mais se torna importante o estudo de biomarcadores envolvidos no envelhecimento cerebral (Gary et al., 2012). Em um dos estudos realizados com cérebros de cães com SDCC (síndrome de disfunção cognitiva canina), foi identificado um biomarcador, chamado Pittsburgh Compound B$P I B$, em maior quantidade na região do cerebelo, o que diferiu dos achados da maioria dos casos em humanos (Fast et al., 2013). Uma pesquisa recente sugere que cães considerados adultos jovens, numa idade em que não é esperada a deposição de amiloide no cérebro e que tinham altos níveis de LCR A42 (beta-amiloide no líquido cefalorraquidiano), demonstraram, em teste-padrão de cognição, deficiência de aprendizagem. Esse resultado sugere que níveis elevados de LCR A-42 são preditivos de deficiências significativas de aprendizagem (Borghysa et al., 2017). Ainda foi observado que a redução dos receptores muscarínicos de alta especificidade nos linfócitos pode ser indicativo de envelhecimento fisiológico, enquanto o aumento nos níveis de receptores muscarínicos de linfócitos de baixa afinidade pode estar relacionado ao declínio cognitivo (Badino et al., 2013).

Com relação às condutas de olfação, vocalização e eliminação de excretas, todos os cães do grupo SAC demonstraram esses comportamentos, pois exploraram a sala por um tempo maior e vocalizaram mais, o que evidencia o comportamento fisiológico dos cães (Siwak et al., 2003) e corrobora o estudo de Martinez et al. (2013), o qual demonstrou que cães jovens e sem sinais de DCC vocalizavam com mais intensidade quando deixados sozinhos. Também durante o teste, os dois cães que foram classificados com DCC (9 e 10) eliminaram excreções, sendo esse parâmetro não constatado nos demais animais; assim, o grupo com DCC diferiu dos demais grupos estudados $(\mathrm{P}=0,0067)$. Isso demonstra que houve uma apresentação de alteração nesse teste, compatível com a perda da cognição dos cães com DCC. Ainda, os cães do grupo DCC urinaram durante os testes, o que se justifica pelo fato de que animais com a síndrome esquecem os comportamentos aprendidos, como comandos básicos e eliminação de excretas, devido às degenerações que ocorrem em nível cerebral (Siwak et al., 2003). 
Em relação ao teste de curiosidade (Fig. 1B), seis cães $(1,2,3,4,5$ e 7$)$ demonstraram interesse pelos objetos e interagiram com eles, sendo esta uma reação considerada esperada, pois os animais exprimem certa curiosidade perante novos objetos (Martinez et al., 2013). Já os cães $6,8,9$ e 10 não interagiram com os brinquedos dispostos na sala. Da mesma forma, a diminuição e a alteração da locomoção, bem como a diminuição dos comportamentos de curiosidade, estão relacionadas à gravidade da DCC (Rosado et al., 2012a).

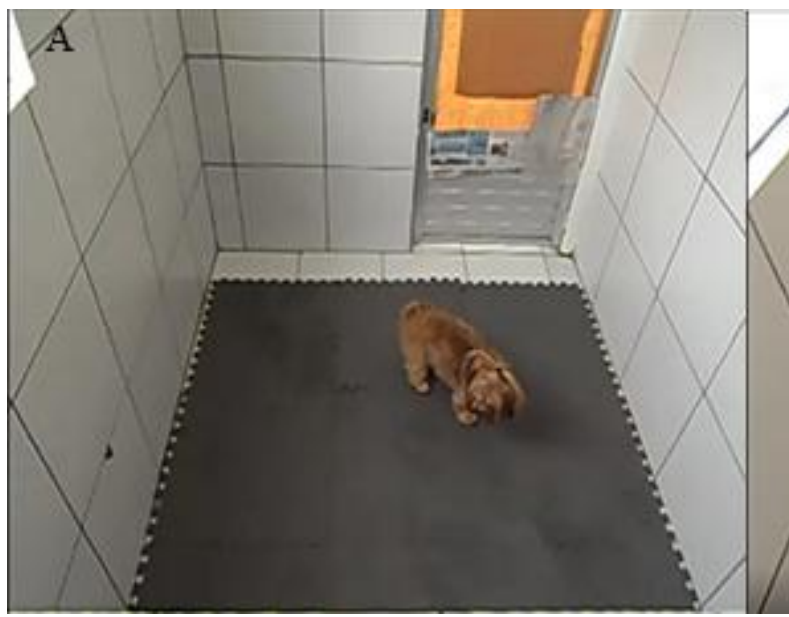

Figura 1. Teste de reatividade: teste open field (A), o cão 9 apresenta marcha estereotipada e não demonstrou interesse em explorar o ambiente; teste de curiosidade (B), o cão 3 não apresenta interação com os três objetos dispostos na sala e demonstra desinteresse por explorar o ambiente.

$\mathrm{Na}$ interação com humanos (Fig. 2C), foi observado que os cães mais idosos procuraram mais a interação (olhar, chegar perto e mover a cauda) com o humano. Quando avaliada a associação do grupo com DCC, os cães 9 e 10 não apresentaram movimento de cauda e aproximação em relação ao humano, sendo constatada, na avaliação estatística, uma diferença com os outros grupos $(\mathrm{P}=0,0357)$, pois os demais apresentavam interação com o humano e movimento de cauda, sendo o fisiológico para animais sem disfunção cognitiva. Em ambos os testes, curiosidade e interação com humanos, foi percebido que cães dos grupos SAC e BL procuraram explorar a sala pelo olfato, ficaram curiosos com os objetos que foram dispostos e interagiram com o humano por meio de olhar e movimento da cauda, o que não foi percebido nos cães do grupo DCC. Estes apresentam um comportamento exploratório menos evidente do que cães sem alterações cognitivas, pois perdem a capacidade espacial (Siwak et al., 2002; Landesberg, 2005). Foi documentado que cães com DCC leve apresentaram maior frequência de alteraçăo na socialização $(70 \%)$, enquanto aqueles com DCC moderada mostraram maior dificuldade de encontrar alimentos (80\%), aqueles com DCC mais severa apresentaram dificuldade de reconhecer o tutor $(90 \%)$ (Salvin et al., 2011).

No teste do espetho'(Fig. 2D), somente o cẩo 5 do grupo $\mathrm{SAC}$, do grupo BL espelho, interá confundindo cão; somente interagiram estatisticame

de interação $(\mathrm{P}=0,0357)$, o que confirma um quadro de perda da cognição. Os cães classificados com DCC apenas ficaram parados diante do espelho a maior parte do tempo, sem interagirem com a própria imagem refletida, demonstrando alteração de comportamento e desorientação espacial, resultado que concorda com estudos realizados por Martinez et al. (2013) em que os cães com DCC passaram a maior parte do tempo apresentando o mesmo comportamento, parados em frente ao espelho, ao contrário dos cães classificados no grupo SAC, que apresentam algum tipo de interação com a própria imagem, uma vez que não são capazes de reconhecer a própria imagem e, sim, interagir como se estivessem observando outro cão (Martinez et al.,2013). 


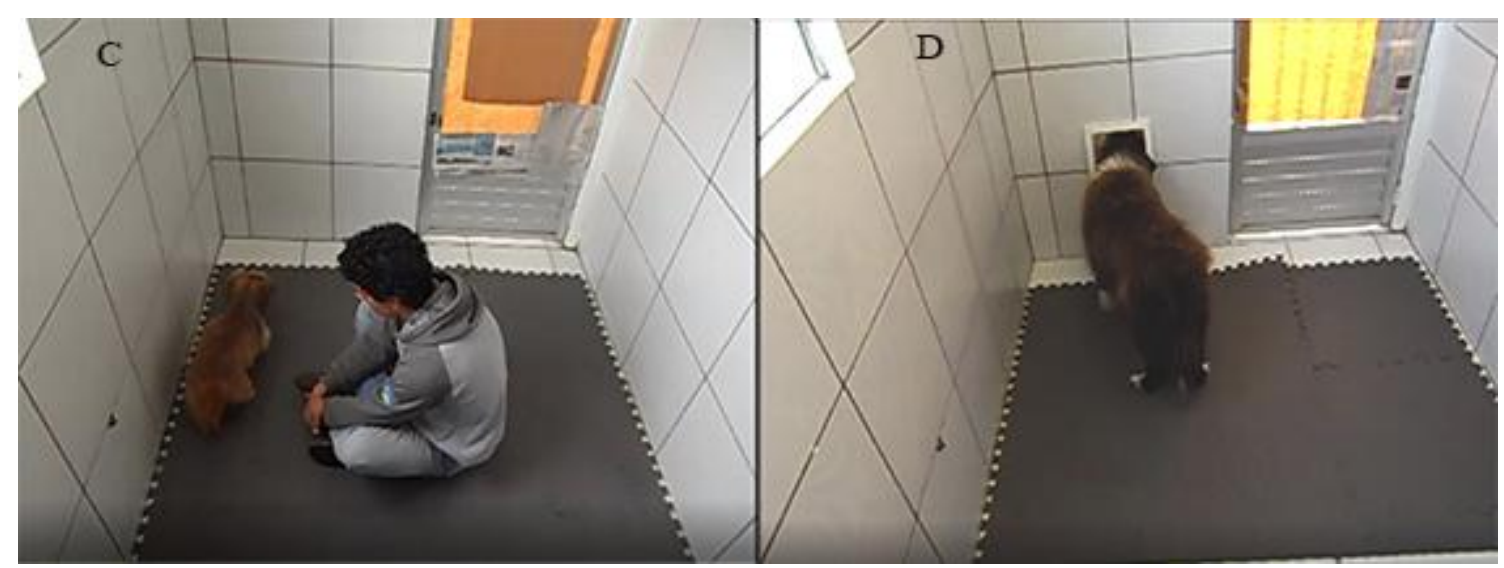

Figura 2. Teste de reatividade: teste de interação com humanos (C), cão 9 não demonstra interesse em interagir com o humano que está sentado no centro da sala; teste do espelho (D), cão 2 está parado em frente ao espelho, interagindo com a própria imagem refletida.

Diante dos resultados apresentados nos cães classificados com DCC, é possível afirmar que muitos desses sinais comportamentais observados durante os testes podem passar despercebidos ou serem considerados como normais pelos tutores. Por isso, é de extrema relevância a realização dos questionários observacionais e dos testes de reatividade para se avaliar o verdadeiro estado cognitivo do paciente idoso e identificar, de maneira precoce, os sinais iniciais da síndrome de disfunção cognitiva canina. Considerando que cães com DCC têm uma alta sobrevida, torna-se necessário o diagnóstico precoce para reduzir a evolução e possibilitar o tratamento adequado, com medicações específicas, dieta rica em antioxidantes e enriquecimento ambiental (Fast et al., 2013). Os medicamentos aprovados que podem ser utilizados em cães são a seligina e a adenosilmetionina (Landesberg, 2005). Já dieta adequada deve ser rica em antioxidantes, sendo um fator decisivo na prevenção de transtornos cognitivos (Katina et al., 2016). Por fim, as estimulações cognitivas devem ser motivadas de forma gradual, pois um ambiente enriquecido aumenta a capacidade mental de cães com demência (Rosado et al., 2012b).

\section{CONCLUSÃO}

Conclui-se que, com o aumento da expectativa de vida canina, o questionário observacional foi um instrumento de triagem para a identificação dos cães classificados com DCC, BL ou SAC, e os testes de reatividade mostrou-se um método inovador para identificar o verdadeiro estado cognitivo dos pacientes idosos.

\section{REFERENCIAS}

BADINO, P.; ODOREA, R.; BERGAMASCO, L. et al. Concentrations of platelet a2adrenoceptors, lymphocyte muscarinic receptors, and blood monoamines in dogs (Canis familiaris) affected by canine cognitive dysfunction syndrome. J. Vet. Behav., v.8, p.146-153, 2013.

BORGHYSA, H.; BROECKA, B.V.; DHUYVETTERA, D. et al. Young to middleaged dogs with high amyloid levels in cerebrospinal fluid are impaired on learning in standard cognition tests. J. Alzheimers Dis., v.56, p.763-774, 2017.

CANINE-Cognitive-Dysfunction-Rating-scaleCCDR. Regenerative Neuroscience Group. 2012. Available in:<http://www.surveygizmo.com/s3/ 1839821/Canine-Cognitive-Dysfunction-Ratingscale-CCDR>. Accessed in: 30 Mar. 2015.

COLLE, M.A.; HAUW, J.J.; CRESPEAU, F. et al. Vascular and parenchymal A $\beta$ deposition in the aging dog: correlation with behavior. Neuro. Biol. Again., v. 21, p. 695-704, 2000.

FAST, R.; RODELL, A.; GJEDDE, A. PiB fails to map amyloid deposits in cerebral cortex of aged dogs with canine cognitive dysfunction. Front. Aging Neurosci., v.5, n.9, p. 822-829, 2013.

FAST, R.; SCHUTT, T.; TOFT, N. et al. An observational study with long-term follow-up of canine cognitive dysfunction: clinical aracteristics, survival, and risk factors. J. Vet. Intern. Med., v.27, p.822-829, 2013. 
GONZÁLEZ-MARTÍNEZ，A.; ROSADO，B.; GARCÍA-BELENGUER, S. et al. Síndrome de disfunción cognitiva en el perro geriátrico. Clin. Vet. Pesqui. Anim., v.32, p.159-167, 2012.

HEAD, E.; LIU, J.; HAGEN, T. M. et al. Oxidative damage increases with age in a canine model of human brain aging. J. Neurochem. v.82, p.375-381, 2002.

HECKLER, M.C.T.; TRANQUILIM, M.V.; SVICERO, D.J. et al. Clinical feasibility of cognitive testing in dogs (Canis lupus familiaris). J.of Veter. Behav., v.9, p.6-12, 2014.

KATINA, S.; FARBAKOVA, J.; MADARI, A. et al. Risk factors for canine cognitive. Vet. J., v.188, p.331-336, 2016.

LANDSBERG, G. Therapeutic agents for the treatment of cognitive dysfunction syndrome in senior dogs. Biol. Psychiatry, v.29, p.471-479, 2005.

LANDSBERG, G.M.; NICHOL, J.; ARAUJO, J.A. Cognitive dysfunction syndrome: a disease of canine and feline brain aging. Vet. Clin. Small Anim., v.42, p.749-768, 2012.

MADARI, A.; FARBAKOVA, J.; KATINA, S. et al. Assessment of severity and progression of canine cognitive dysfunction syndrome using the canine dementia scale (cades). Appl. Anim. Behav. Sci., v.171, p.138-145, 2015.

MARTÍNEZ, A.G.; ROSADO, B.; PESINI, P. et al. Effect of age and severity of cognitive dysfunction on two simple tasks in pet dogs. Vet. J., v.198, p.176-181, 2013.

OSELLA, M.C.; RE, G.; ODORE, R. et al. Canine cognitive dysfunction syndrome: prevalence, clinical signs and treatment with a neuroprotective nutraceutical. J. Appl. Behav. Sci., v.105, p.297-310, 2007.

PACKER, R. M. A.; MCGREEVY, P.D.; SALVIN, H.E. et al. Cognitive dysfunction in naturally occurring canine idiopathic epilepsy. Jour. Plos One. v.13 (2) p. 1-20, 2018.

PINEDA, S.; OLIVARES, A.; MAS, B. et al. Cognitive dysfunction syndrome: updated behavioral and clinical evaluations as a tool to evaluate the well-being of aging dogs. Arch. Med. Vet., v.46, p.1-12, 2014.
ROFINA, J.E.; VAN EDEREN, A.M.; TOUSSAINT, M.J.M.; SECRÈVE, M. et al. Cognitive disturbances in old dogs suffering from the canine counterpart of Alzheimer's disease. Brain Res., v.1069, p.216-226, 2006.

ROSADO, B.; MARTÍNEZ, A.G.; PESINI, P. et al. Effect of age and severity of cognitive dysfunction on spontaneous activity in pet dogs Part 1: Locomotor and exploratory behaviour. Vet. J., v.194, p.189-195, 2012a.

ROSADO, B.; MARTÍNEZ, A.G.; PESINI, P. et al. Effect of age and severity of cognitive dysfunction on spontaneous activity in pet dogs Part 2: Social responsiveness. Vet. J., v.194, p.196-201, 2012b.

SALVIN, H.E.; MCGREEVY, P.D.; SACHDEV, P.S. et al. The canine cognitive dysfunction rating scale (CCDR): a data-driven and ecologically relevant assessment tool. Vet. J., v.188, p.331-336, 2011.

SCHUTT, T.; TOFT, N.; BERENDT, M. A comparison of two screening questionnaires for clinical assessment of canine cognitive dysfunction. J. Vet. Behav., v.10, p.452-458, 2015.

SIWAK, C.T.; MURPHEY, H.L.; MUGGENBURG, B.A. et al. Age-dependent decline in locomotor activity in dogs is environment specific. Physiol. Behav., v.75, p.65-70, 2002

SIWAK, C.T.; TAPP, P.D.; ZICKER, S. C. et al. Locomotor activity rhythms in dogs vary with age and cognitive status. Behav. Neurosci., v.117, p.813-824, 2003.

TAPP, P.D.; SIWAK, C.T.; GAO, F.Q. et al. Frontal lobe volume, function, and beta-amyloid pathology in a canine model of aging. $J$. Neurosci., v.24, p.8205-8213, 2004.

WALLIS, L.; VIRÁNYI, Z.; MULLER, C.A. et al. Aging effects on discrimination learning, logical reasoning and memory in pet dogs. Spring, v.38, p.6, 2016.

YU, C.H.; SONG, G.S.; YHEE, J.H. et al. Histopathological and immunohistochemical comparison of the brain of human patients with Alzheimer's disease and the brain of aged dogs with cognitive dysfunction. J. Comp. Pathol., v.145, p.45-58, 2011. 